\title{
Anabases
}

ANABASES Traditions et réceptions de l'Antiquité

$17 \mid 2013$

Varia

\section{Suzanne L. MARCHAND, German Orientalism in the Age of Empire. Religion, Race, and Scholarship}

\section{Corinne Bonnet}

\section{OpenEdition}

\section{Journals}

Édition électronique

URL : http://journals.openedition.org/anabases/4280

DOI : $10.4000 /$ anabases. 4280

ISSN : 2256-9421

\section{Éditeur}

E.R.A.S.M.E.

\section{Édition imprimée}

Date de publication : 1 mars 2013

Pagination : 292-294

ISSN : 1774-4296

\section{Référence électronique}

Corinne Bonnet, «Suzanne L. marchand, German Orientalism in the Age of Empire. Religion, Race, and Scholarship », Anabases [En ligne], 17 | 2013, mis en ligne le 01 avril 2013, consulté le 22 septembre 2020. URL : http://journals.openedition.org/anabases/4280 ; DOI : https://doi.org/10.4000/anabases 4280

Ce document a été généré automatiquement le 22 septembre 2020

(c) Anabases 


\title{
Suzanne L. MARCHAND, German Orientalism in the Age of Empire. Religion, Race, and Scholarship
}

\author{
Corinne Bonnet
}

\section{RÉFÉRENCE}

Suzanne L. MARCHAND, German Orientalism in the Age of Empire. Religion, Race, and Scholarship, German Historical Institute (Washington D.C.), Cambridge, Cambridge University Press, 2009, 560 p. 48 livres / isbn 9780521169073.

1 En dix chapitres nourris par une érudition exceptionnelle, Suzanne Marchand nous livre ici une histoire culturelle de l'Allemagne vue à travers le prisme de l'orientalisme, science importante, parfois fondatrice, mais souvent ambivalente pour diverses raisons. Cette science engage, en effet, dans son existence même et dans la construction de ses objets, comme l'a (trop ?) bien montré Edward Said, une certaine relation de domination de l'Europe sur les contrées situées au-delà de la Méditerranée. Elle englobe la «Terre promise ", donc l'étude de la Bible, qui pose des problèmes «idéologiques » particuliers. Elle prétend cerner l'aube de la civilisation et le berceau du monothéisme, autant de paramètres qui pèsent lourd dans son développement et son positionnement. Si le livre de S. Marchand est particulièrement bienvenu, c'est que Said lui-même avait avancé l'idée, depuis contestée sans avoir cependant fait l'objet d'une contre-analyse approfondie, que l'Allemagne ne pouvait, en ces matières, être purement et simplement assimilée à la France et à l'Angleterre, puissances conquérantes et colonisatrices en Orient. La science allemande aurait, selon Said, développé, à l'égard de l'Orient, une approche différente, ne relevant pas d'une logique impérialiste. S. Marchand s'empare de cette prétendue exception allemande en s'affranchissant du cadre trop contraignant de l'orientalisme saïdien qui fond toutes les relations Orient-Occident dans le moule de 
l'impérialisme. Ici l'approche se veut pragmatique, au plus près des réalités du terrain de la science, et affiche une certaine méfiance à l'égard de la méthode de l'analyse du discours (discourse analysis) qui peut déboucher sur une vision trop standardisée des pratiques intellectuelles. Il s'agit plutôt de faire émerger la variété des positionnements à l'égard de l'Orient et de son passé de la part des savants allemands, eux-mêmes issus de milieux culturels très diversifiés, loin de toute approche binaire et de l'obsession du pouvoir dérivant de la connaissance ou s'appuyant sur elle. C'est pourquoi, au cœur de l'enquête, S. Marchand place « a set of practices » qui prennent l'Orient comme objet et qui engagent des «savants » issus de la théologie, de l'archéologie, de la linguistique comparée, des lettres classiques, mais qui mobilisent aussi des institutions, des revues, des sociétés savantes...

2 C'est dans la longue durée que S; Marchand inscrit une enquête qu'elle entame avec les Lumières, lorsque l'orientalisme n'est pas encore à proprement parler une discipline. C'est surtout l'Ancien Testament qui donne alors accès à l'Orient, mais les évolutions géopolitiques font que l'on prête de plus en plus d'attention à l'Empire ottoman, puis à l'Inde avec la découverte de l'Avesta publiée en 1771 en France et en 1776-1783 en Allemagne, voire à l'Extrême-Orient. On entre déjà alors dans une sorte de première Renaissance orientale dont S. Marchand souligne les spécificités en Allemagne, un pays où les activités culturelles étaient encore intimement liées aux institutions cléricales. De grandes personnalités déjà se dégagent, comme J.G. Herder et J.D. Michalis qui croisent le fer autour de l'Ancien Testament, source encore hégémonique dont on tente de s'affranchir progressivement. Rapidement se pose aussi la question du rapport de l'Orient à la Grèce, matrice par excellence de la Kultur, avec laquelle l'Allemagne entretient une affinité très élective.

3 En plus de 500 pages d'une grande densité, S. Marchand nous propose une galerie de portraits vraiment impressionnante (Schlegel, Ritter, Creuzer, Richthofen, Lagarde, Nöldeke, Wellhausen, Oppenheim, Erman, Meyer, Deussen, Goldziher, Becker...) qui permet de restituer le tissu scientifique allemand dans toute sa complexité, avec l'émergence progressive de sous-domaines comme la Semitistik, l'Indogermanistik, la Judaistik, l'Arabistik... Le traitement de l'ensemble de la matière fait émerger avec force la place primordiale qui revient aux questions religieuses et linguistiques dans l'étude de l'Orient. C'est aussi par ce biais qu'en France, Ernest Renan en vient à aborder la question des races et de l'esprit qui les caractérise, le conduisant à formuler des jugements sur le poids des races dans les développements historiques. La " passion des races » trouve aussi sa place dans les très belles analyses de S. Marchand, au chapitre 7, où elle montre comment la science orientaliste allemande construit et convoque le couple Aryens-Sémites. Outre une approche diachronique dans la longue durée, qui va des Lumières au nazisme dans l'Épilogue en passant par le Furor orientalis qui précède la Première Guerre mondiale, on touche à plusieurs problématiques transversales, comme celle de l'art oriental ou de la place du comparatisme dans l'étude des civilisations orientales (avec la question de la primauté de la Bible ou de Babylone; ou celle du rapprochement entre Bouddha et Jésus).

4 C'est une somme que S. Marchand a réalisée et qu'elle met à notre disposition. Pour l'écrire, elle a eu recours à une impressionnante panoplie de sources: publications, archives, correspondances... Elle débouche sur des conclusions solides et nuancées concernant l'implication malgré tout indéniable des orientalistes dans la politique impérialiste de l'Allemagne (à la façon de Said, mais avec mille nuances 
supplémentaires) et dans les théories raciales, puis racistes. Les progrès intellectuels accomplis par les orientalistes allemands sont nombreux et considérables; ils ont contribué à l'ouverture de l'Europe à d'autres cultures et ont permis de remettre progressivement, mais pas totalement en question le «miracle grec» d'une part, le «miracle biblique» de l'autre. En certains points, la synthèse de S. Marchand se recoupe avec le bel ouvrage de Sabine Mangold, Eine "weltbürgerliche Wissenschaft ». Die deutsche Orientalistik im 19. Jahrhundert, Stuttgart, Franz Steiner Verlag, 2004. On signalera aussi, sur un sujet proche, le volume d'Ursula Wokoeck, German Orientalism : The Study of the Middle East and Islam from 1800 to 1945, Londres, Routledge, 2009. Enfin, rappelons que S. Marchand a obtenu, en 2010, pour ce livre marquant le George L. Mosse Prize de l'American Historical Association, qu'on ne peut que féliciter pour son choix.

\section{AUTEURS}

\section{CORINNE BONNET}

Université de Toulouse (UTM)

cbonnet@univ-tlse2.fr 